\title{
Analysis of the impact of COVID-19 on the coupling of the material flow and capital flow in a closed-loop supply chain
}

\author{
Duan, W. ${ }^{a}, M a, H^{a}{ }^{\text {, Xu, D.S. }}{ }^{\text {a,b, }{ }^{*}}$ \\ ${ }^{a}$ School of Economics and Management, Inner Mongolia University of Technology, Hohhot, P.R. China \\ ${ }^{b}$ Inner Mongolia Modern Logistics and Supply Chain Management Research Center, Hohhot, P.R. China
}

\begin{abstract}
A B S T R A C T
The complex and changeable external social and economic environment has a significant impact on the sustainable development of the closed-loop supply chain. In particular, the occurrence of uncertain emergencies increases the risk of interruption of the closed-loop supply chain, making it insufficient to analyze its complex changes from the perspective of material flow alone. Based on this analysis, the paper constructs a closed-loop supply chain material flow and capital flow coupling system composed of manufacturers, sellers and recyclers to explore the impact of material flow sudden interruption on the closed-loop supply chain system when an uncertain emergency occurs. In this paper, based on the closed-loop supply chain system coupled with logistics and capital flow, a system dynamics simulation model was established by using Vensim simulation software to analyze the impact of COVID-19 epidemic on manufacturers, sellers and recyclers under five scenarios. The results show that when COVID-19 outbreaks occur, the material flow of each main enterprise in the closed-loop supply chain is more easily influenced than the capital flow. At the same time, it can be found that the recyclers in the main enterprises of the closed-loop supply chain are more easily influenced by the material flow. The model constructed in this paper has applicability and can be used for related studies of closed-loop supply chain under other emergencies, but the scene design should be carried out according to the characteristics of emergencies themselves.
\end{abstract}

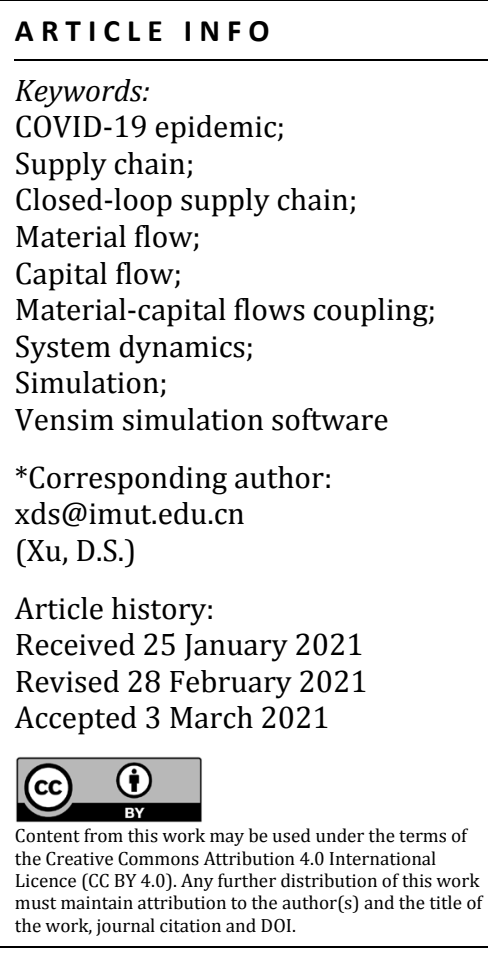

\section{References}

[1] Kim, Y.-W., Chang, T.-W., Park, J. (2017). Gen2 RFID-based system framework for resource circulation in closedloop supply chains, Sustainability, Vol. 9, No. 11, doi :10.3390/su9111995.

[2] Coenen, J., van der Heijden, R., van Riel, A.C.R., Allard C.R. (2019). Making a transition toward more mature closed-loop supply chain management under deep uncertainty and dynamic complexity: A methodology, Sustainability, Vol. 11, No. 8, doi: 10.3390/su11082318.

[3] Aminipour, A., Bahroun, Z., Hariga, M. (2021). Cyclic manufacturing and remanufacturing in a closed-loop supply chain, Sustainable Production and Consumption, Vol. 25, 43-59, doi: 10.1016/spc202008002.

[4] Peng, H., Shen, N., Liao, H., Xue, H., Wang, Q. (2020). Uncertainty factors, methods, and solutions of closed-loop supply chain - A review for current situation and future prospects, Journal of Cleaner Production, Vol. 254, Article No. 120032, doi: 10.1016/clepro120032.

[5] Islam, M.T., Huda, N. (2018). Reverse logistics and closed-loop supply chain of waste electrical and electronic equipment (WEEE)/ E-waste: A comprehensive literature review, Resources, Conservation and Recycling, Vol. 137, 48-75, doi: 10.1016/ resconrec05026. 
[6] Delgoshaei, A., Farhadi, M., Hanjani Esmaeili, S., Delgoshaei, A., Mirzazadeh, A. (2019). A new method for distributing and transporting of fashion goods in a closed-loop supply chain in the presence of market uncertainty, Industrial Engineering \& Management Systems, Vol. 18. No. 4, 825-844, doi: 10.7232/iems.2019.18.4.825.

[7] Zhang, X. (2019). Research on coping strategies of supply disruption under stochastic demand, Academic Journal of Business \& Management, Vol. 1, No. 1,99-112.

[8] Morakabatchiankar, S., Hjaila, K., Mele, F.D., Graells, M., Espuña, A. (2018). Economic and environmental benefits of waste-based energy closed-loop integration in process industries under uncertainty, Computer Aided Chemical Engineering, Vol. 43, 501-506, doi: 10.1016/B978-0-444-64235-6.50089-9.

[9] Cao, J., Yan, Y., Wang, L., Chen, X., Zhang, X., Zhou, G. (2020). Emergency strategies of closed-loop supply chain with single retailer recycling under demand disruptions, Discrete Dynamics in Nature and Society, Vol. 2020, Article ID 9817641, doi: 10.1155/2020/9817641.

[10] Liao, H., Deng, Q., Wang, Y. (2017). Optimal acquisition and production policy for end-of-life engineering machinery recovering in a joint manufacturing /remanufacturing system under uncertainties in procurement and demand, Sustainability, Vol. 9, No. 3, Article No. 338, doi: 10.3390/su9030338.

[11] Almaraj, I.I., Trafalis, T.B. (2020). Affinely adjustable robust optimization under dynamic uncertainty set for a novel robust closed-loop supply chain, Computers \& Industrial Engineering, Vol. 145, Article No. 106521, doi: $10.1016 /$ j.cie.2020.106521.

[12] Vahdani, B., Ahmadzadeh, E. (2019). Designing a realistic ICT closed loop supply chain network with integrated decisions under uncertain demand and lead time, Knowledge-Based Systems, Vol. 179, 34-54, doi: 10.1016/ knosys.05003.

[13] Chen, X., Li, K., Wang, F., Li, X. (2020). Optimal production, pricing and government subsidy policies for a closed loop supply chain with uncertain returns, Journal of Industrial and Management Optimization, Vol. 16, No. 3, 1389-1414, doi: 10.3934/jimo.2019008.

[14] Olivares-Aguila, J., ElMaraghy, W. (2020). System dynamics modelling for supply chain disruptions, International Journal of Production Research, Vol 58, 1-19, doi: 10.1080/00207543.2020.1725171.

[15] Chen, L., Dui, H., Zhang, C. (2020). A resilience measure for supply chain systems considering the interruption with the cyber-physical systems, Reliability Engineering \& System Safety, Vol. 199, Article No. 106869, doi: 10.1016/ress.106869.

[16] Sánchez-Ramírez, C., Ramos-Hernández, R., Mendoza Fong, J.R., Alor-Hernández, G., García-Alcaraz, J.L. (2020). A system dynamics model to evaluate the impact of production process disruption on order shipping, Applied Sciences, Vol. 10, No. 1, Article No. 208, doi: 10.3390/app10010208.

[17] Shao, L., Jin, S. (2020). Resilience assessment of the lithium supply chain in China under impact of new energy vehicles and supply interruption, Journal of Cleaner Production, Vol. 252, Article No. 119624, doi: 10.1016/ jclepro.19624.

[18] Wang, Y., Wang, J., Wang, X. (2020). COVID-19, supply chain disruption and China's hog market: A dynamic analysis, China Agricultural Economic Review, Vol. 12, No. 3, 427-443, doi: 10.1108/CAER-04-2020-0053.

[19] Kim, J., Chung, B.D., Kang, Y., Jeong, B. (2018). Robust optimization model for closed-loop supply chain planning under reverse logistics flow and demand uncertainty, Journal of Cleaner Production, Vol. 196, 1314-1328, doi: 10.1016/j.jclepro.2018.06.157.

[20] Hassanpour, A., Bagherinejad, J., Bashiri, M. (2019). A robust leader follower approach for closed loop supply chain network design considering returns quality levels, Computers \& Industrial Engineering, Vol. 136, 293-304, doi: 10.1016/j.cie.2019.07.031.

[21] Fazli-Khalaf, M., Chaharsooghi, S.K., Pishvaee, M.S. (2019). A new robust possibilistic programming model for reliable supply chain network design: A case study of lead-acid battery supply chain, RAIRO-Operations Research, Vol. 53, No. 5, 1489-1512, doi: 10.1051/ro/2018073.

[22] Polo, A., Peña, N., Muñoz, D., Cañón, A., Escobar, J.W. (2019). Robust design of a closed-loop supply chain under uncertainty conditions integrating financial criteria, Omega. Vol. 88, 110-132, doi: 10.1016/j.omega.2018.09.003.

[23] Abdolazimi, O., Salehi Esfandarani, M., Salehi, M., Shishebori, D. (2020). Robust design of a multi-objective closed-loop supply chain by integrating on-time delivery, cost, and environmental aspects, case study of a Tire factory, Journal of Cleaner Production, Vol. 264, Article No. 121566, doi: 10.1016/j.jclepro.2020.121566.

[24] Gholizadeh, H., Tajdin, A., Javadian, N. (2020). A closed-loop supply chain robust optimization for disposable appliances, Neural Computing and Applications, Vol. 32, No. 8, 3967-3985, doi: 10.1007/s00521-018-3847-9.

[25] Mohammed, F., Hassan, A., Selim, S.Z. (2019). Carbon market sensitive robust optimization model for closed loop supply chain network design under uncertainty, Journal of Physics: Conference Series, Vol. 1150, Article No. 012009, doi: 10.1088/1742-6596/1150/1/012009.

[26] Nayeri, S., Paydar, M.M., Asadi-Gangraj, E., Emami, S. (2020). Multi objective fuzzy robust optimization approach to sustainable closed-loop supply chain network design, Computers \& Industrial Engineering, Vol. 148, Article No. 106716, doi: 10.1016/i.cie.2020.106716.

[27] Tan, Y., Guo, C. (2019). Research on two-way logistics operation with uncertain recycling quality in government multi-policy environment, Sustainability, Vol. 11, No. 3, Article No. 882, doi: 10.3390/su11030882.

[28] Hosseini-Motlagh, S.-M., Nami, N., Farshadfar, Z. (2020). Collection disruption management and channel coordination in a socially concerned closed-loop supply chain: A game theory approach, Journal of Cleaner Production, Vol. 276, Article No. 124173, doi: 10.1016/i.jclepro.2020.124173.

[29] Jin, M., Zhou, Y. (2020). Does the remanufactured product deserve the same warranty as the new one in a closedloop supply chain?, Journal of Cleaner Production, Vol. 262, Article No. 121430, doi: 10.1016/i.jclepro.2020. 121430. 
[30] Jauhari, W.A., Adam, N.A.F.P., Rosyidi, C.N., Nyoman Pujawan, I., Shah, N.H. (2020). A closed-loop supply chain model with rework, waste disposal, and carbon emissions, Operations Research Perspectives, Vol. 7, Article No. 100155, doi: 10.1016/j.orp.2020.100155.

[31] Yu, H., Solvang, W.D. (2016). A stochastic programming approach with improved multi-criteria scenario-based solution method for sustainable reverse logistics design of waste electrical and electronic equipment (WEEE), Sustainability, Vol. 8, No. 12, Article No. 1331, doi: 10.3390/su8121331.

[32] Pourjavad, E., Mayorga, R.V. (2018). Optimization of a sustainable closed loop supply chain network design under uncertainty using multi-objective evolutionary algorithms, Advances in Production Engineering \& Management, Vol. 13, No. 2, 216-228, doi: 10.14743/apem2018.2.286.

[33] Dehghan, E., Amiri, M., Shafiei Nikabadi, M., Jabbarzadeh, A. (2019). Novel robust fuzzy programming for closedloop supply chain network design under hybrid uncertainty, Journal of Intelligent \&Fuzzy Systems, Vol. 37, No. 5, 6457-6470, doi: $10.3233 /$ IIFS-18117.

[34] Ghomi-Avili, M., Khosrojerdi, A., Tavakkoli-Moghaddam, R. (2019). A multi-objective model for the closed-loop supply chain network design with a price-dependent demand, shortage and disruption, Journal of Intelligent \& Fuzzy Systems, Vol. 36, No. 6, 5261-5272, doi: 10.3233/IIFS-181051.

[35] Fakhrzad, M.B., Goodarzian, F. (2019). A Fuzzy multi-objective programming approach to develop a green closed-loop supply chain network design problem under uncertainty: Modifications of imperialist competitive algorithm, RAIRO Operations Research, Vol. 53, No. 3, 963-990, doi: 10.1051/ro/2019018.

[36] Santander, P., Cruz Sanchez, F.A., Boudaoud, H., Camargo, M. (2020). Closed loop supply chain network for local and distributed plastic recycling for 3D printing: A MILP-based optimization approach, Resources, Conservation and Recycling, Vol. 154, Article No. 104531, doi: 10.1016/i.resconrec.2019.104531.

[37] Zhao, R., Liu, Y., Zhang, Z., Guo, S., Tseng, M.-L., Wu, K.-J. (2018). Enhancing eco-efficiency of agro-products' closed-loop supply chain under the belt and road initiatives: A system dynamics approach, Sustainability, Vol. 10, No. 3, Article No. 668, doi: 10.3390/su10030668.

[38] Goltsos, T.E., Ponte, B., Wang, S., Liu, Y., Naim, M.M., Syntetos, A.A. (2019). The boomerang returns? Accounting for the impact of uncertainties on the dynamics of remanufacturing systems, International Journal of Production Research, Vol. 57, No. 23, 7361-7394, doi: 10.1080/00207543.2018.1510191.

[39] Zhao, J., Sun, N. (2020). Government subsidies-based profits distribution pattern analysis in closed-loop supply chain using game theory, Neural Computing \& Applications, Vol. 32, No. 6, 1715-1724, doi: 10.1007/s00521-019$\underline{04245-2}$

[40] Miao, S., Liu, D., Ma, J., Tian, F. (2020). System dynamics modelling of mixed recycling mode based on contract: A case study of online and offline recycling of E-waste in China, Mathematical and Computer Modelling of Dynamical Systems, Vol. 26, No. 3, 234-252, doi: 10.1080/13873954.2020.1762096.

[41] Xue, R., Zhang, F., Tian, F. (2018). A system dynamics model to evaluate effects of retailer-led recycling based on dual chains competition: A case of e-waste in China, Sustainability, Vol. 10, No. 10, Article No. 3391, doi: $10.3390 /$ su10103391.

[42] Huang, M., Yi, P., Shi, T., Guo, L. (2018). A modal interval based method for dynamic decision model considering uncertain quality of used products in remanufacturing, Journal of Intelligent Manufacturing, Vol. 29, No. 4, 925935, doi: $10.1007 / \mathrm{s} 10845-015-1151-4$.

[43] Sahebjamnia, N., Fathollahi-Fard, A.M., Hajiaghaei-Keshteli, M. (2018). Sustainable tire closed-loop supply chain network design: Hybrid metaheuristic algorithms for large-scale networks, Journal of Cleaner Production, Vol. 196, 273-296, doi: 10.1016/j.jclepro.2018.05.245.

[44] Zarbakhshnia, N., Kannan, D., Kiani Mavi, R, Soleimani, H. (2020). A novel sustainable multi-objective optimization model for forward and reverse logistics system under demand uncertainty, Annals of Operations Research, Vol. 295, No. 2, 843-880, doi: 10.1007/s10479-020-03744-z.

[45] Feitó-Cespón, M., Costa, Y., Pishvaee, M.S., Cespón-Castro, R. (2021). A fuzzy inference based scenario building in two-stage optimization framework for sustainable recycling supply chain redesign, Expert Systems with Applications, Vol. 165, Article No. 113906, doi: 10.1016/j.eswa.2020.113906.

[46] Zic, J., Zic, S. (2020). Multi-criteria decision making in supply chain management based on inventory levels, environmental impact and costs, Advances in Production Engineering \& Management, Vol. 15, No. 2, 151-163, doi: 10.14743/apem2020.2.355

[47] Freile, A.J., Mula, J., Campuzano-Bolarin, F. (2020). Integrating inventory and transport capacity planning in a food supply chain, International Journal of Simulation Modelling, Vol. 19, No. 3, 434-445, doi: 10.2507/IJSIMM19$\underline{3-523}$.

[48] Buschiazzo, M., Mula, J., Campuzano-Bolarin, F. (2020). Simulation optimization for the inventory management of healthcare supplies, International Journal of Simulation Modelling, Vol. 19, No. 2, 255-266, doi: 10.2507/IJSIMM19-2-514.

[49] $\mathrm{Hu}, \mathrm{H} ., \mathrm{Shi}, \mathrm{L} ., \mathrm{Ma}, \mathrm{H} ., \mathrm{Ran}, \mathrm{B}$. (2017). Stability of the supply chain based on disruption classification, Tehnički Vjesnik - Technical Gazette, Vol. 24, No. 4, 1187-1195, doi: 10.17559/TV-20170723084826.

[50] Babaeinesami, A., Tohidi, H., Seyedaliakbar, S.M. (2020). A closed loop Stackelberg game in multi-product supply chain considering information security: A case study, Advances in Production Engineering \& Management, Vol. 15, No. 2, 233-246, doi: 10.14743/apem2020.2.361.

[51] Zhang, Y.C., Feng, Y., Zhou, J.H., Zhang, S.X. (2018). Simulation and optimization of contract coordination model of closed-loop supply chain quality control based on system dynamics, Systems Engineering, Vol. 36, No. 3, 105-112. 


\title{
Analiza vpliva COVID-19 na povezana toka materiala in kapitala v krožni dobavni verigi
}

\author{
Duan, W. ${ }^{\mathrm{a}}, \mathrm{Ma}, \mathrm{H.}^{\mathrm{a}},{ }^{\mathrm{X}} \mathrm{Xu}, \mathrm{D}$. S. $^{\mathrm{a}, \mathrm{b},{ }^{*}}$ \\ aschool of Economics and Management, Inner Mongolia University of Technology, Hohhot, P.R. China \\ bInner Mongolia Modern Logistics and Supply Chain Management Research Center, Hohhot, P.R. China
}

\section{POVZETEK}

Kompleksno in spremenljivo družbeno in ekonomsko okolje pomembno vpliva na trajnostni razvoj krožne dobavne verige. Zlasti pojav izrednih razmer predstavlja pomembno negotovost in poveča tveganje za prekinitev krožne dobavne verige, zaradi česar je kompleksne spremembe krožne dobavne verige nezadostno analizirati samo z vidika toka materiala. Članek opisuje analizo toka materiala in toka kapitala v krožni dobavni verigi, ki ga sestavljajo proizvajalci, prodajalci in predelovalci. Cilj je raziskati vpliv nenadne prekinitve toka materiala zaradi pojava izrednih razmer na delovanje krožne dobavne verige. $V$ raziskavi je bil na podlagi sistema krožne dobavne verige, skupaj z logistiko in tokom kapitala, z uporabo simulacijske programske opreme Vensim postavljen simulacijski model sistemske dinamike za analizo vpliva epidemije COVID-19 na proizvajalce, prodajalce in predelovalce po petih scenarijih. Rezultati kažejo, da je ob izbruhih COVID-19 v krožni dobavni verigi v glavnih podjetjih lažje vplivati na tok materiala kot na kapitalski tok. Obenem je mogoče ugotoviti, da so predelovalci v glavnih podjetjih v krožni dobavni verigi pod vplivom toka materiala. Model, predstavljen v tem članku, je uporaben in se lahko uporablja za sorodne raziskave krožne dobavne verige v drugačnih izrednih razmerah, vendar je treba okoliščine prilagoditi značilnostmi spremenjenih izrednih razmer.

\author{
PODATKI O ČLANKU \\ Ključne besede: \\ Epidemija COVID-19; \\ Dobavna veriga; \\ Krožna dobavna veriga; \\ Tok materiala; \\ Tok kapitala; \\ Povezani materialno-kapitalski \\ tokovi; \\ Sistemska dinamika; \\ Simulacija; \\ Programska oprema za simulacijo \\ Vensim \\ * Kontaktna oseba: \\ xds@imut.edu.cn \\ (Xu, D.S.) \\ Zgodovina članka: \\ Prejet 25. januarja 2021 \\ Popravljen 28. februarja 2021 \\ Sprejet 3. marca 2021

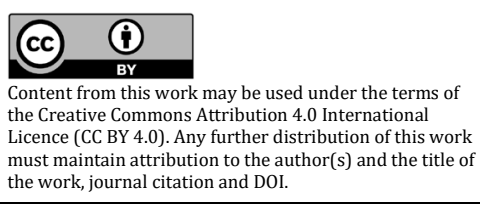

